\title{
A novel approach to design and fabrication of thermo-acoustic refrigerator using high amplitude sound waves
}

\author{
Viswa Mohan Pedagopu ${ }^{1}$ Khannaji Pattapu ${ }^{2}$ \\ 1. Assistant Professor, Department of Mechanical Engineering, Shoolini University, Bajhol. Solan, Himachal \\ Pradesh, India \\ 2. Bachelor of Technology in Mechanical Engineering, kothagudem, AP.
}

\begin{abstract}
The basic object of this paper is compression of refrigerant by the consumption of high amplitude sound waves that results the generation thermal Energy. For generation of sound waves a loud speaker (or common audio speaker) has been employed for creating high amplitude sound waves which compresses refrigerant (helium) by the well Known thermodynamics process of heat absorption. A Thermo acoustic refrigerator is fabricated with a cooler and loud speaker. The acoustic power produced by a loud speaker as a sound waves reaches to heat the pump. The cooler works on a reverse Carnot cycle by incorporating a compact acoustic network to create the travelling wave phasing necessary to operate. As the acoustic wave propagates temperature difference occurs at the ends of stack, heat and cold is trap by the heat exchangers regenerator from a cold heat exchanger to an ambient one. Instead of using co-axial topology network, the toroidal is used. The design, construction and performance measurement of the cooler are also discussed. Finally, at $8^{0} \mathrm{C}$ a coefficient of performance 7.0 is achieved.
\end{abstract}

Key words: Thermo Acoustic Refrigeration, toroidal, high amplitude sound waves, heat exchanger.

\section{Introduction}

Over the past two decades, physicists and engineers have been working on a class of heat engines and compression-driven refrigerators, the area where the oscillating pistons, oil seals or lubricants are not required. Due to the globalization the reduction of cost for any process has become mandatory and even some times inevitable. This paper deals with thermo acoustic devices take advantage of sound waves reverberating within them to convert a temperature differential into mechanical energy and vice-versa. The basic entity of this paper is generation of heat energy by using high amplitude sound waves by heat absorption technique. A loud speaker has been engaged to create high amplitude sound waves that can compress refrigerant by heat absorption. The researches have subjugated the fact that sound waves travel by compressing and expanding the gas generates some thermal energy. If the said waves travel through a tube or pipe and of course, a stack of plates is placed at the right place within the said tube due to the bouncing sound waves around causes the generation of a temperature gradient. It is observed that some plates in the stack hotter and other colder. This condition is possible to make a refrigerator.

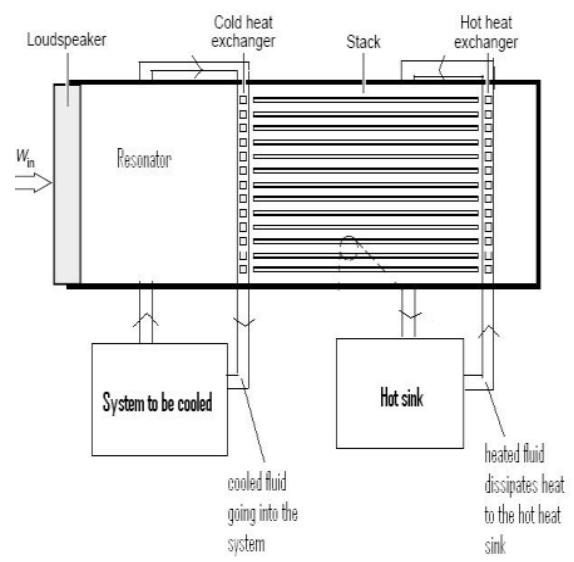

Figure 1. Temperature variation in device

Figure 1. An overview of thermo -Acoustic refrigeration system

Thermo acoustic refrigerator is a special kind of device that uses energy of sound waves or acoustic energy to pump heat from low temperature reservoir to a high temperature reservoir. The source of acoustic energy is called the 'driver' which can be a loudspeaker. The driver emits sound waves in a long hollow tube filled with gas at high pressure. This long hollow tube is called as 'resonance tube' or simply 'resonator'. The frequency of 
the driver and the length of the resonator are chosen so as to get a standing sound wave in the resonator. A solid porous material like a stack of parallel plates is kept in the path of sound waves in the resonator. Due to thermo acoustic effect (which will be explained in detail in the animation), heat starts to flow from one end of stack to the other. One end starts to heat up while other starts to cool down. By controlling temperature of hot side of stack (by removing heat by means of a heat exchanger), the cold end of stack can be made to cool down to lower and lower temperatures. A refrigeration load can then be applied at the cold end by means of a heat exchanger.

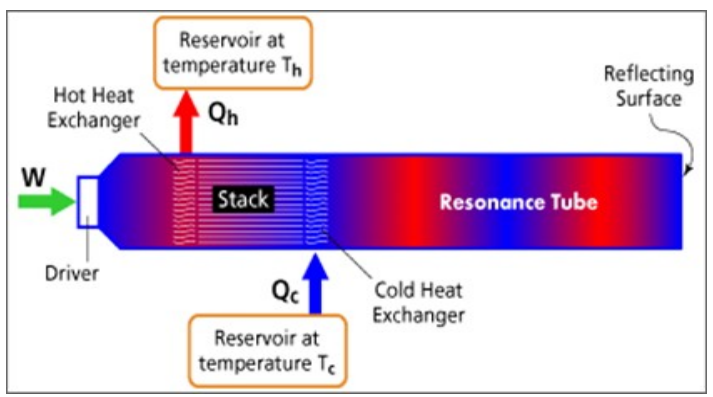

Figure 2: Temperature variation in device

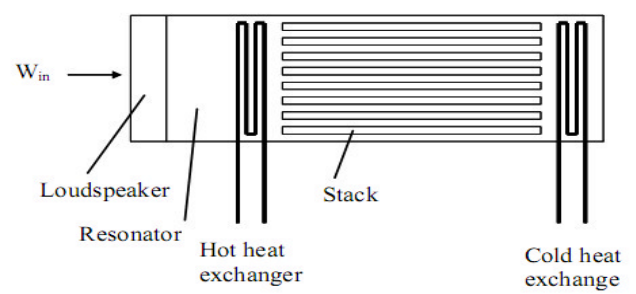

Figure 3. The basic principle of thermo -Acoustic refrigeration system

Thermo acoustics combines the branches of acoustics and thermodynamics together to move heat by using sound. While acoustics is primarily concerned with the macroscopic effects of sound transfer like coupled pressure and motion oscillations, thermo acoustics focuses on the microscopic temperature oscillations that accompany these pressure changes. Thermo acoustics takes advantage of these pressure oscillations to move heat on a macroscopic level. This results in a large temperature difference between the hot and cold sides of the device and causes refrigeration.

\section{Construction And Working MAIN PARTS OF TAR}

Two main parts are in the TAR

1. Loud speaker.2. Resonator. 3. Stack

4. Heat exchangers.

5. Helium gas.

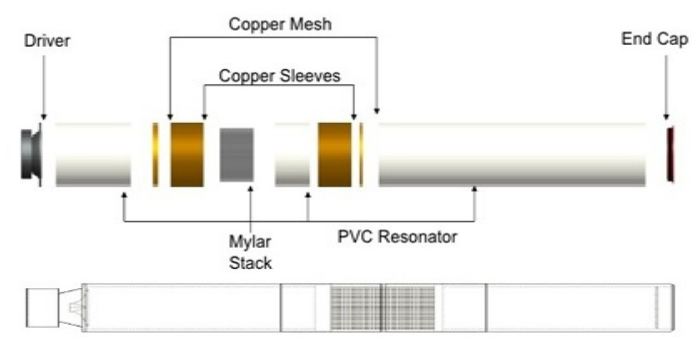

Figure 4. The working principle of thermo -Acoustic refrigeration system 


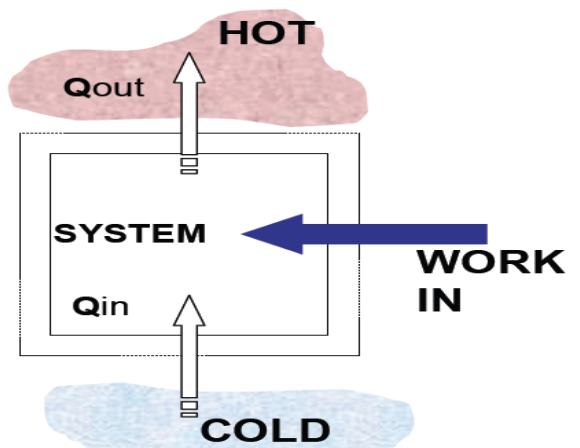

Figure 5. Thermodynamic System

A loudspeaker (or "speaker") is an electro acoustic transducer that produces sound in response to an electrical audio signal input. A coil of wire is attached to the apex of large paper cone and placed between the poles of a permanent magnet.

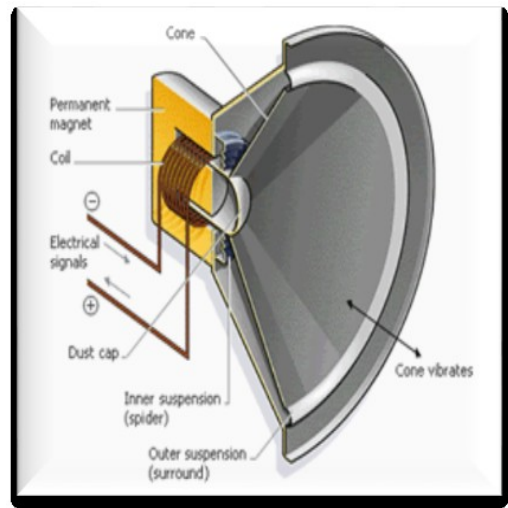

Figure 6. Loud speakers

When an electrical current passes through the coil, it sets up a magnetic field .This produces vibrations in the cone by which sound is emitted. A loud speaker is employed for to generate a standing Acoustic wave.

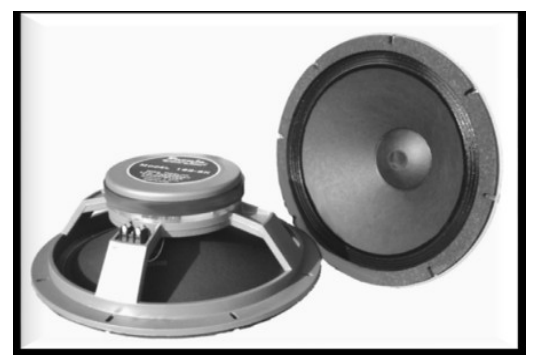

Figure 7. Sound boxes for generation of sound waves

A resonant tube is an organ pipe which is a cylindrical tube having an air column. If the resonator has fixed or closed boundaries at both ends, the amplitude of the wave is forced to zero and velocity nodes are formed at both ends. These nodes occur at multiples of $\lambda / 2(\lambda / 2, \lambda, 3 \lambda / 2,2 \lambda$, and so on) and therefore these resonators are known as half wavelength resonators.

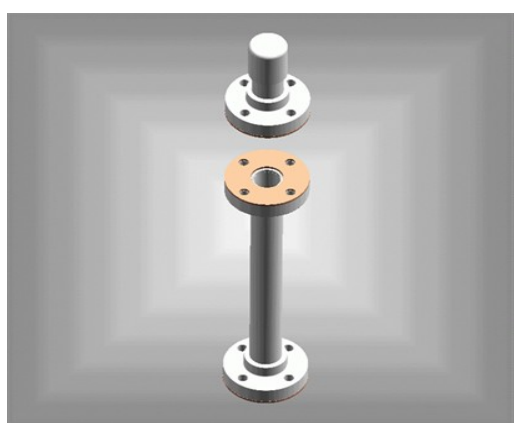

Figure 8. A compression wave in an organ pipe 
When a compression wave is sent through an organ pipe, the wave gets reflected at the ends of the pipe these incident and reflected waves which are in a same frequency, travelling in opposite directions are super imposed along the length of the pipe and form longitudinal stationary waves. As diaphragm vibrates the alternate Compressions and expansions occurs throughout the Gas medium in the resonant column.

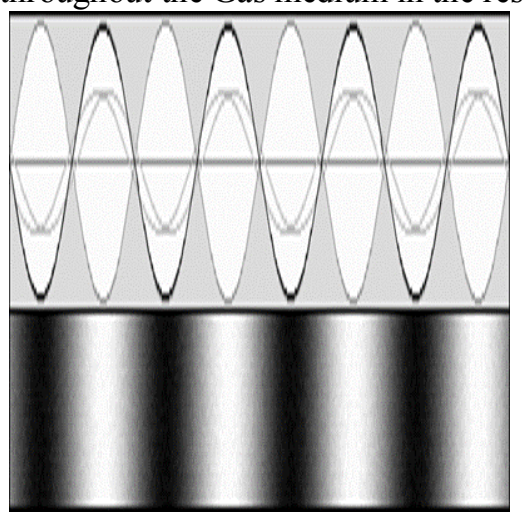

Figure 9. Generation of longitudinal stationary waves

Heat exchangers are devices used to transfer heat energy from one fluid to another. They are two heat exchangers:

1. Hot heat exchanger and

2. Cold heat exchanger

Heat exchanger is a copper plate fitted in a copper sleeve. The plate is punched with a number of holes. The diameter of the holes matches with the gap between stack plates.

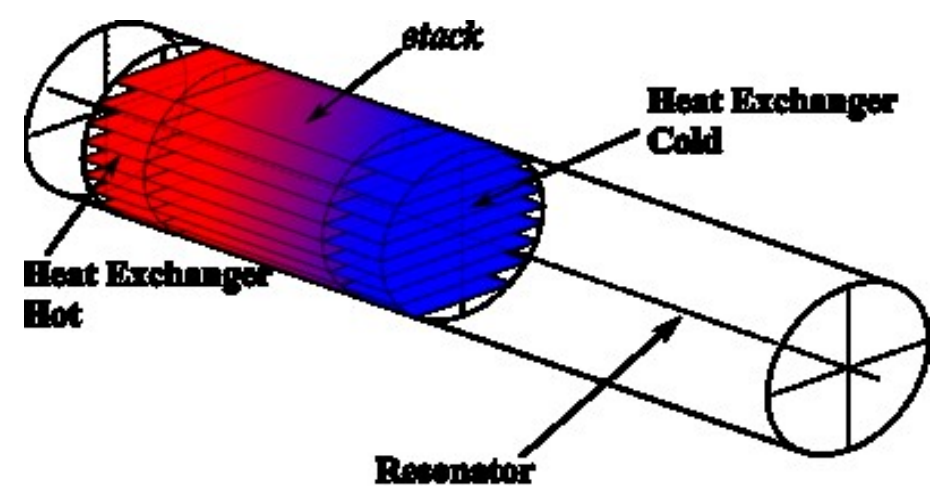

Figure 10. Working of resonator

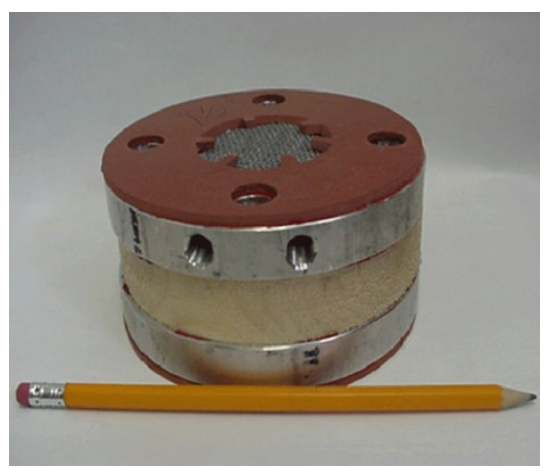

He-Gas is a light weight inert gas with small atomic size. With usage of helium gas the gap between the stack plates will be minimized to a large extent. Because with the less atomic size vanderwaal's forces are weaker with respect to other noble gases. Therefore kinematic viscosity is very low. So, the molecules are free to vibrate even in a small gaps resulting high utilization of gas molecules for to participate in heat transfer. 
Figure 11. Helium gas cylinder

It is called as regenerator. The stack consists of a large number of closely spaced surfaces that are aligned parallel to the to the resonator tube. In a usual resonator tube, heat transfer occurs between the walls of cylinder and the gas.

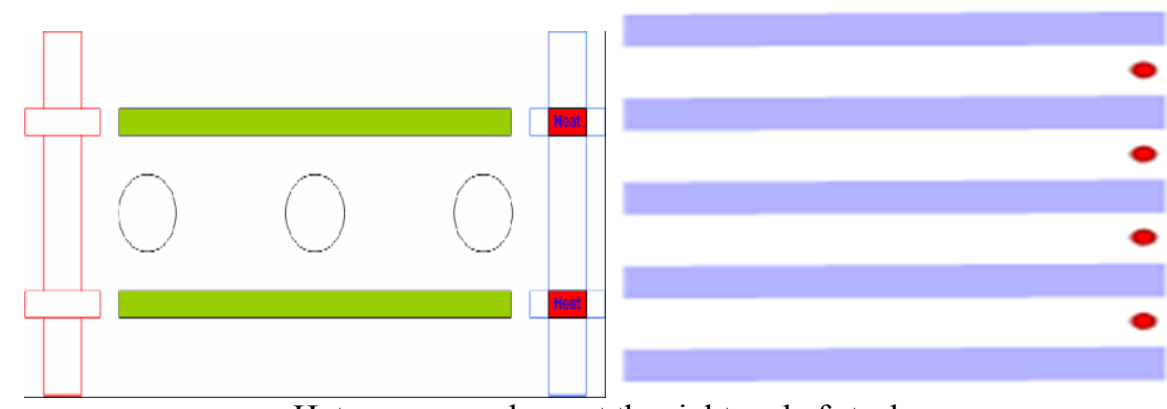

Hot compressed gas at the right end of stack

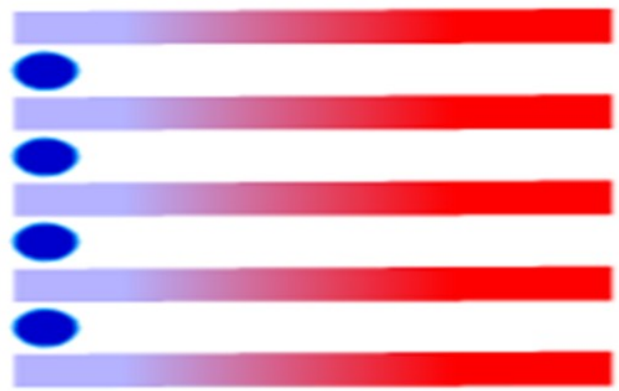

Gas expands while moving to left and cools

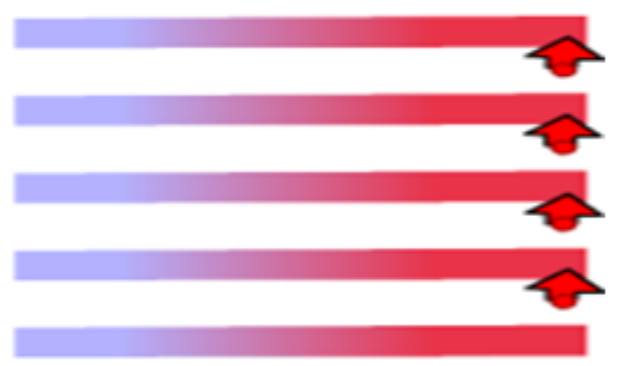

Heat loss to stack. Stack temperature rises.

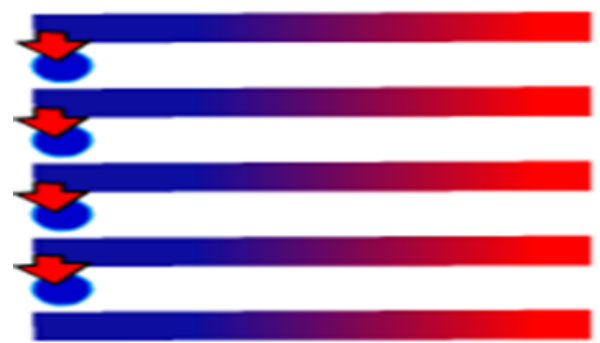

Cold gas takes heat from stack. Stack is colder

If the holes are too narrow, the stack will be difficult to fabricate, and the viscous properties of the air will make it difficult to transmit sound through the stack. If the walls are too far apart, then less air will be able to transfer 
heat to the walls of the stack, resulting in lower efficiency. The different materials Paper, Aluminium, Lexan Foam

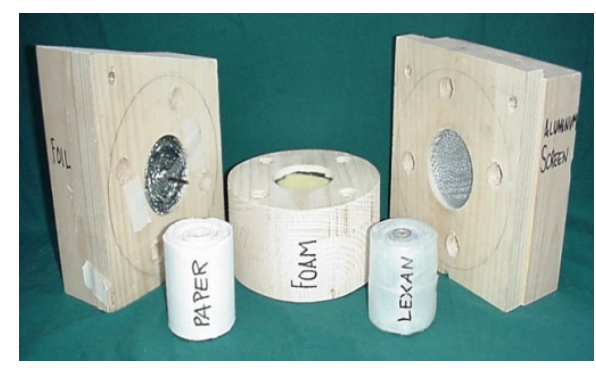

\section{Applications}

Liquefaction of natural gas: Burning natural gas in a thermo acoustic engine generates acoustic energy. This acoustic energy is used in a thermo acoustic heat pump to liquefy natural gas.

Chip cooling: In this case a piezoelectric element generates the sound wave. A thermo acoustic heat pump cools the chip.

Electronic equipment cooling on naval ships: In this application, a speaker generates sound waves. Again a thermo acoustic pump is used to provide the cooling.

Electricity from sunlight: Concentrated thermal solar energy generates an acoustic wave in a heated thermo acoustic engine. A linear motor generates electricity from this.

Upgrading industrial waste heat: Acoustic energy is created by means of industrial waste heat in a thermo acoustic engine. In a thermo acoustic heat pump this acoustic energy is used to upgrade the same waste heat to a useful temperature level.
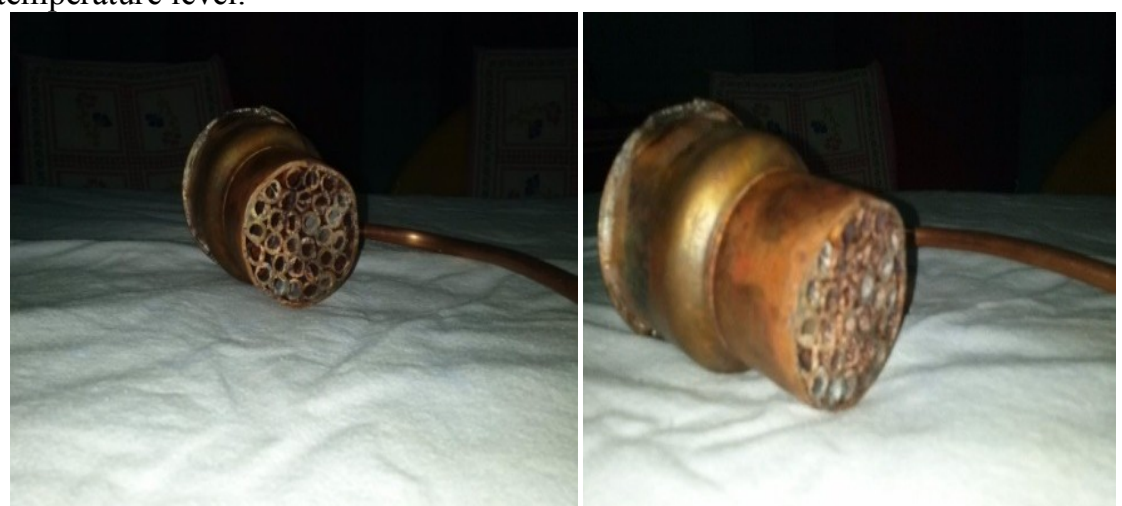

Figure 12. Copper tubes of cold side of stack which function as cold heat exchanger other end is closed by a copper cap.

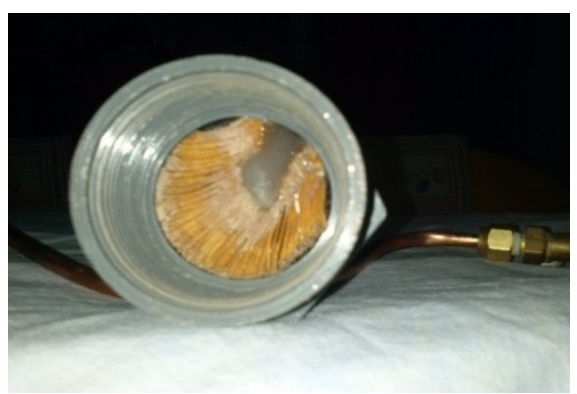

Figure 13. High quality filter paper rolled stack (coil) 


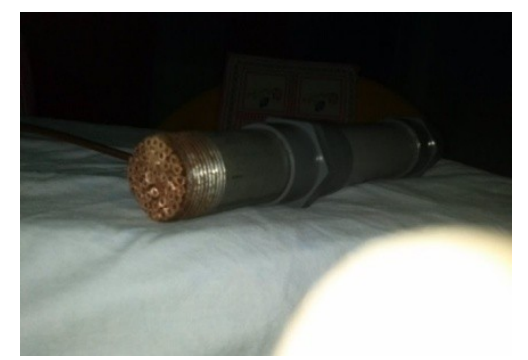

Figure 14. Copper tubes with iron sleeve attached to hot side of stack

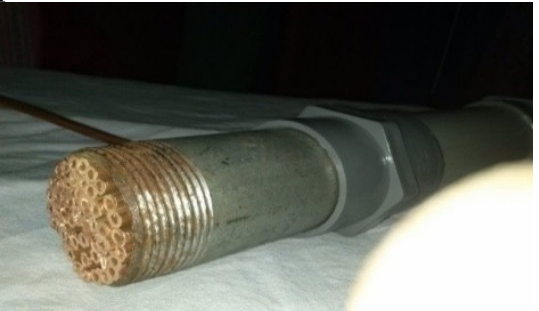

Figure 15. Copper tubes of hot heat exchanger
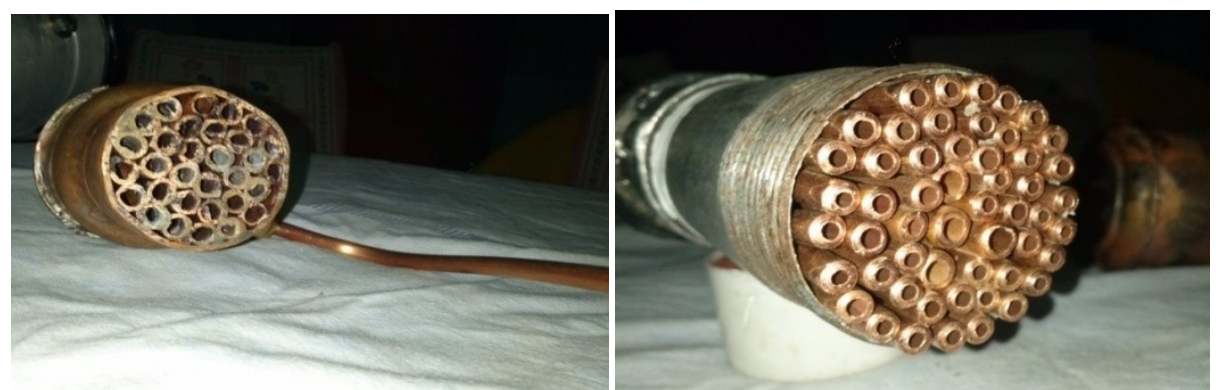

Figure 16. Copper tubes arranged parallel to the wave propagation

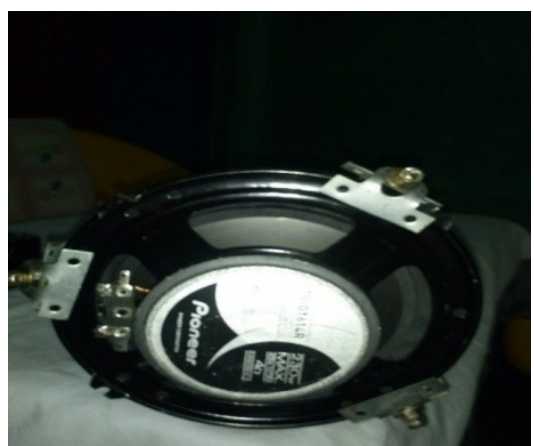

Figure 17. Loud speaker to generate acoustic power

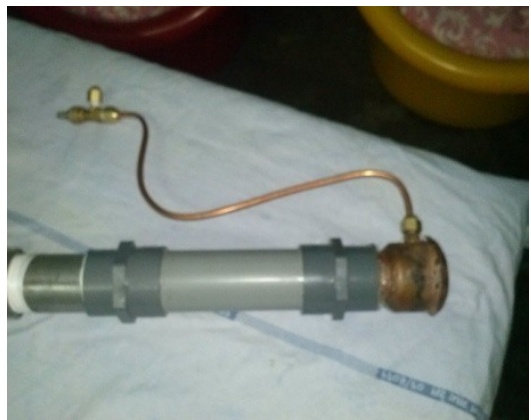

Figure 18. Hot and cold heat exchangers are thermally separated by PVC pipe.

Working: Thermo acoustics combines the branches of acoustics and thermodynamics together to move heat by using sound. While acoustics is primarily concerned with the macroscopic effects of sound transfer like coupled 
pressure and motion oscillations, thermo acoustics focuses on the microscopic temperature oscillations that accompany these pressure changes. Thermo acoustics takes advantage of these pressure oscillations to move heat on a macroscopic level. This results in a large temperature difference between the hot and cold sides of the device and causes refrigeration. The most important piece of a thermo acoustic device is the stack. The stack consists of a large number of closely spaced surfaces that are aligned parallel to the to the resonator tube. The purpose of the stack is to provide a medium for heat transfer as the sound wave oscillates through the resonator tube. A functional cross section of the stack we used is shown in figure 6. In typical standing wave devices, the temperature differences occur over too small of an area to be noticeable.

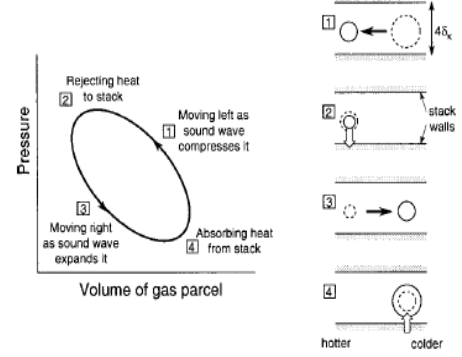

Figure No. Cyclic process

Thermo acoustic refrigerator cycle. The left end is towards the closed end of the resonator tube.

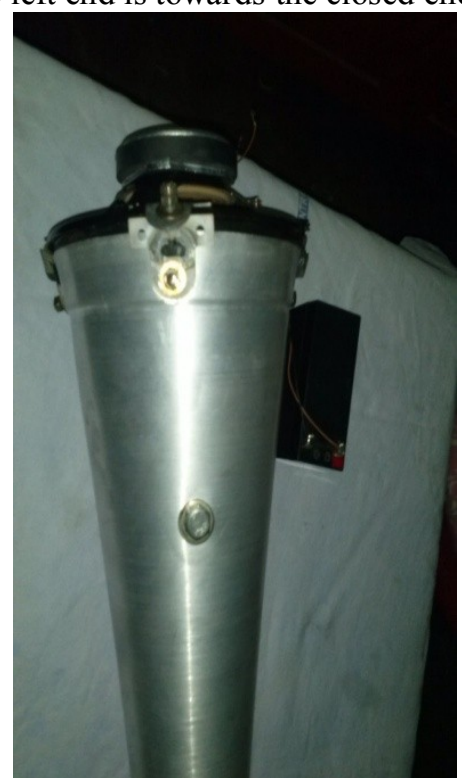

Figure: A conical section for intensifying of sound wave
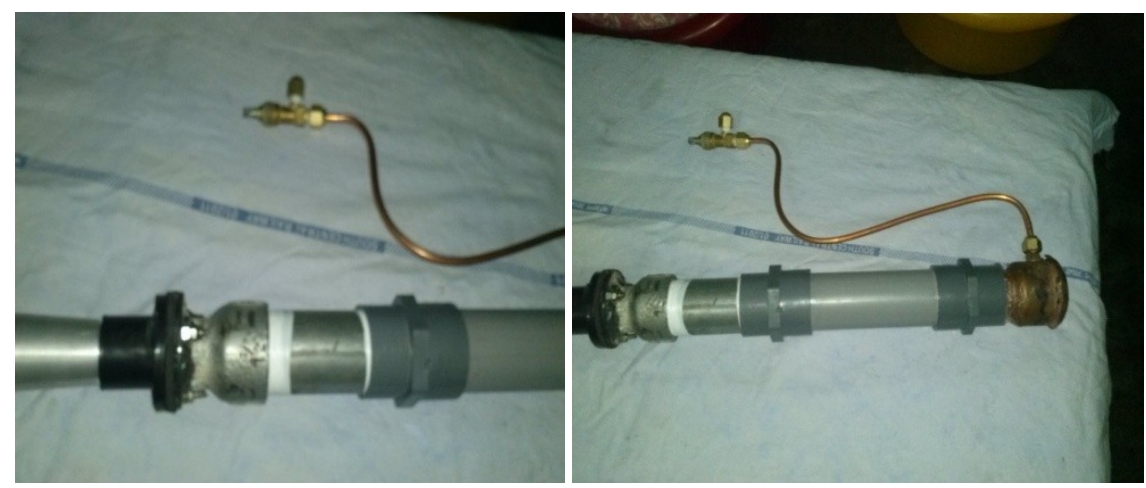

Figure: Helium gas with valve 


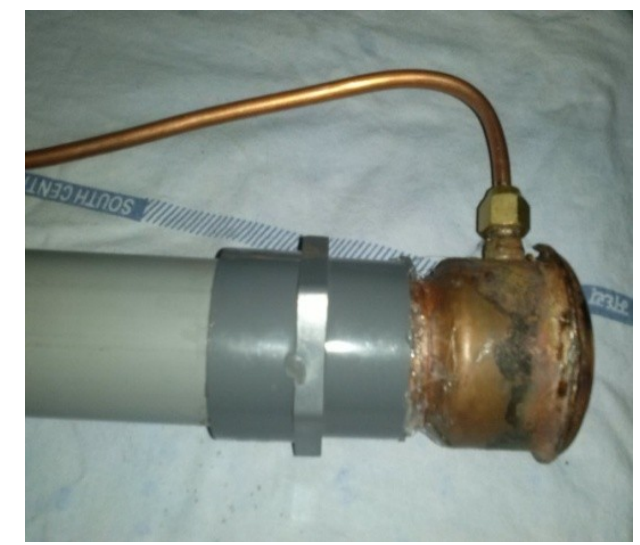

Figure: Inlet portion of Helium gas with valve

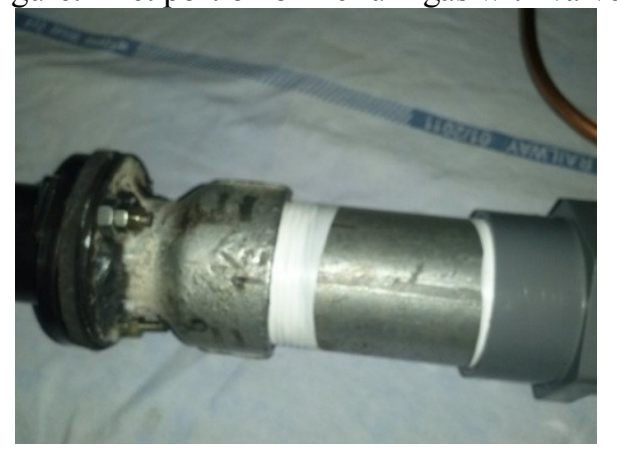

Figure: Hot heat exchanger sealed with glue and Teflon tape.

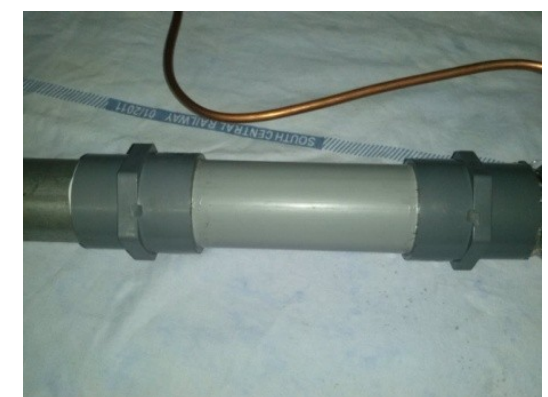

Figure: Stack enclosed by a PVC tube

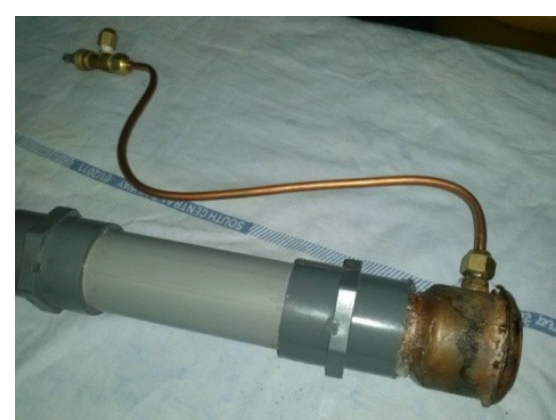

Figure: Copper pipe arrangement projected outside for gas filling and evacuating provided by a valve

Advantages of TAR: No moving parts for the process, so very reliable and a long life span. Environmentally friendly working medium (air, noble gas). The use of air or noble gas as working medium offers a large window of applications because there are no phase transitions. Use of simple materials with no special requirements, which are commercially available in large quantities and therefore relatively cheap. On the same technology base a large variety of applications can be covered. 


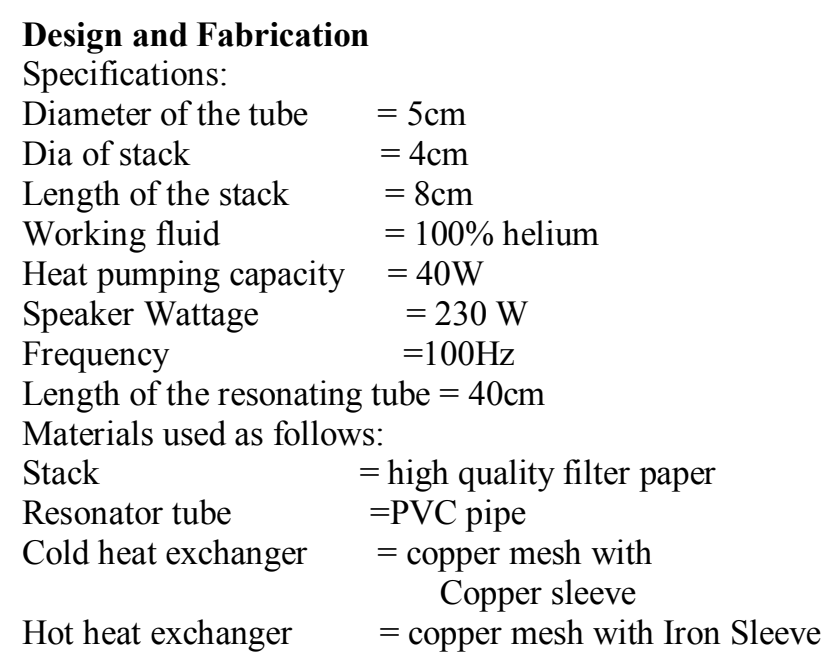

\section{Result:}

A loud speaker operated by a micro controller and relay set up gives pulses as per the programmed frequency. Therefore we can able to maintain a constant standing wave with fixed wavelength. A conical shaped aluminum concentrator having dia D1, D2 intensifies the acoustic waves with the loud speaker sealed at larger diameter and other end having a flange to couple with hot heat exchanger flange a leak proof gasket is fixed between the flanges, which are made up of plastic. Rest of the equipment having high quality filter paper rolled stack glued to a PVC pipe to which both the ends of the pipe are threaded to hold hot and cold heat exchangers.

Hot heat exchanger consisted of iron sleeve; a no of copper tubes having small dia occupies the entire diameter of the sleeve. One end of the sleeve fixed to a slack end. The copper tubes are arranged parallel to the wave propagation. Cold heat exchanger is also same as hot heat exchanger but consists of copper cap having a copper pipe arrangement projected outside the gas filling and evacuating provided with a valve. The ends of all the copper pipes which are near by the stack ends are in contact with stack.

The Cooling effect from the system is found and then the theoretical Coefficient of performance is measured finally, at $8^{\circ} \mathrm{C}$ a coefficient of performance 7.0 is achieved.

\section{Conclusions}

Thermo acoustic engines and refrigerators were already being considered a few years ago for specialized applications, where their simplicity, lack of lubrication and sliding seals, and their use of environmentally harmless working fluids were adequate compensation for their lower efficiencies. In future let us hope these thermo acoustic devices which promise to improve everyone's standard of living while helping to protect the planet might soon take over other costly, less durable and polluting engines and pumps. The latest achievements of the former are certainly encouraging, but there are still much left to be done.

\section{References}

[1]. Heat and mass transfer data book by C.P.kodandaram and S.subramanyan

[2]. Thermo dynamics by R.S.kurmi ,R.K rajput

[3]. Refrigeration and air conditioning by domkundwar, Arora

[4]. http://hyperphysics.phy-astr.gsu.edu/hbase/thermo/carnot.html.

[5]. Daniel A. Russell and Pontus Weibull, "Tabletop thermo acoustic refrigerator for demonstrations," Am. J. Phys. 70 (12), December 2002.

[6]. G. W. Swift, "Thermo acoustic engines and refrigerators," Phys. Today 48, 22-28 (1995).

[7]. http://www.howstuffworks.com/stirling-engine.htm.

[8]. http://en.wikipedia.org/wiki/Carnot_cycle.

[9]. Chilling at Ben \& Jerry's: Cleaner, Greener.” Ken Brown. Available:

[10]. http://www.thermoacousticscorp.com/news/index.cfm/ID/4.htm. 17 July 2006.

[11]. S. L. Garrett and S. Backhaus, "The power of sound," Am. Sci. 88, 516- 525 (2000). 